\title{
Distribution of water around the nucleus of comet 67P/Churyumov-Gerasimenko at 3.4 AU from the Sun as seen by the MIRO instrument on Rosetta
}

\author{
N. Biver ${ }^{1}$, M. Hofstadter ${ }^{2}$, S. Gulkis ${ }^{2}$, D. Bockelée-Morvan ${ }^{1}$, M. Choukroun ${ }^{2}$, E. Lellouch ${ }^{1}$, F. P. Schloerb ${ }^{3}$, L. Rezac ${ }^{4}$, \\ W. H. Ip ${ }^{5}$, C. Jarchow ${ }^{4}$, P. Hartogh ${ }^{4}$, S. Lee ${ }^{2}$, P. von Allmen ${ }^{2}$, J. Crovisier ${ }^{1}$, C. Leyrat ${ }^{1}$, and P. Encrenaz ${ }^{6}$ \\ ${ }^{1}$ LESIA, Observatoire de Paris, CNRS, UPMC, Université Paris-Diderot, 5 place Jules Janssen, 92195 Meudon, France \\ e-mail: nicolas.biver@obspm.fr \\ 2 JPL/California Institute of Technology, 4800 Oak Grove Dr., Pasadena, CA 91109, USA \\ 3 University of Massachusetts, 619 Lederle Graduate Research Tower, Amherst, MA, USA \\ 4 Max Planck Institut für Sonnensystemforschung, Justus-von-Liebig-Weg 3, 37077 Göttingen, Germany \\ 5 National Central University, Jhongli, 32001 Taoyuan City, Taiwan \\ ${ }^{6}$ LERMA, Observatoire de Paris, PSL Research University, UPMC, CNRS, 61 avenue de l'Observatoire, 75014 Paris, France
}

Received 13 March 2015 / Accepted 2 May 2015

\section{ABSTRACT}

\begin{abstract}
The Microwave Instrument on the Rosetta Orbiter (MIRO) has been observing the coma of comet 67P/Churyumov-Gerasimenko almost continuously since June 2014 at wavelengths near $0.53 \mathrm{~mm}$. We present here a map of the water column density in the inner coma (within $3 \mathrm{~km}$ from nucleus center) when the comet was at $3.4 \mathrm{AU}$ from the Sun. Based on the analysis of the $\mathrm{H}_{2}^{16} \mathrm{O}$ and $\mathrm{H}_{2}^{18} \mathrm{O}(110-101)$ lines, we find that the column density can vary by two orders of magnitude in this region. The highest column density is observed in a narrow region on the dayside, close to the neck and north pole rotation axis of the nucleus, while the lowest column density is seen against the nightside of the nucleus where outgassing seems to be very low. We estimate that the outgassing pattern can be represented by a Gaussian distribution in a solid angle with $F W H M \approx 80^{\circ}$.
\end{abstract}

Key words. comets: individual: 67P/Churyumov-Gerasimenko - radio lines: planetary systems - submillimeter: planetary systems comets: general

\section{Introduction}

Comets are the most pristine remnants of the formation of the solar system 4.6 billion years ago. Understanding their composition and the sublimation process of their ices as they heat up approaching the Sun are some of the main objectives of the Rosetta mission to the comet 67P/Churyumov-Gerasimenko (67P). Comet 67P is a Jupiter-family comet that will reach perihelion on 13 August 2015 at 1.24 AU from the Sun. After a ten-year-long journey in the solar system, the Rosetta spacecraft of the European Space Agency reached (at a distance to the comet of $<100 \mathrm{~km}$ ) comet $67 \mathrm{P}$ on 6 August 2015, at $3.6 \mathrm{AU}$ from the Sun. The Microwave Instrument for the Rosetta Orbiter (MIRO) is a submillimeter radiometer equipped with two continuum channels at 188 and $562 \mathrm{GHz}$ and a high spectral resolution heterodyne spectrometer working in frequency-switching mode that targets dedicated $\mathrm{H}_{2} \mathrm{O}, \mathrm{CH}_{3} \mathrm{OH}, \mathrm{CO}$, and $\mathrm{NH}_{3}$ lines in the 548-579 GHz range (Gulkis et al. 2007).

Of particular importance is water, the ice most abundant in the solar nebula and in comets. MIRO observes the (110-101) water line at $557 \mathrm{GHz}$ that connects the first ortho levels of $\mathrm{H}_{2}^{16} \mathrm{O}$. This line cannot be observed from the ground because of atmospheric opacity. It was first observed in comets from Earth orbit with dedicated satellites: the Submillimeter Wave Astronomy Satellite (SWAS; Neufeld et al. 2000), the Odin satellite (Lecacheux et al. 2003), and the Herschel Space Observatory (Hartogh et al. 2010, 2011).
These distant observations, however, only provide a global view of the coma as seen from one direction; at best, a map of the outer coma. Onboard Rosetta, the MIRO instrument (Gulkis et al. 2007) offers the possibility of dissecting the inner coma and exploring how it is structured according to activity regions. The high spectral resolution $\left(44 \mathrm{kHz}\right.$ or $20 \mathrm{~ms}^{-1}$ ) of the Chirp Transform Spectrometer (CTS; Hartogh \& Hartmann 1990) provides strong constraints on the velocity field of the gas.

MIRO first detected the $\mathrm{H}_{2}^{16} \mathrm{O}$ emission from comet 67P on 6 June 2014 at 3.9 AU from the Sun (Gulkis et al. 2015) and has been continuously monitoring the water emission from the comet since early July 2014. In this paper we concentrate on one map of the $\mathrm{H}_{2}^{16} \mathrm{O}$ (110-101) line obtained in September 2014 at 3.4 AU from the Sun and describe what it implies about the spatial distribution of water relative to the nucleus and anisotropy in the outgassing.

\section{Observations}

Two periods on 7 September 2014 were dedicated to mapping the nucleus and close coma of comet 67P with MIRO. Since it is a fixed single-beam instrument with a Gaussian sensitivity with a half-power beam width of $7.5^{\prime}$ at $562 \mathrm{GHz}$, it is necessary to slew the spacecraft across the target to map it. The scanning scheme was set up such that the Rosetta spacecraft nominal pointing axis ( $z$-axis) was slewed at a rate of $48^{\prime} / \mathrm{min}$ (about 3 -submillimeter beams during each single $30 \mathrm{~s}$ integration) along 

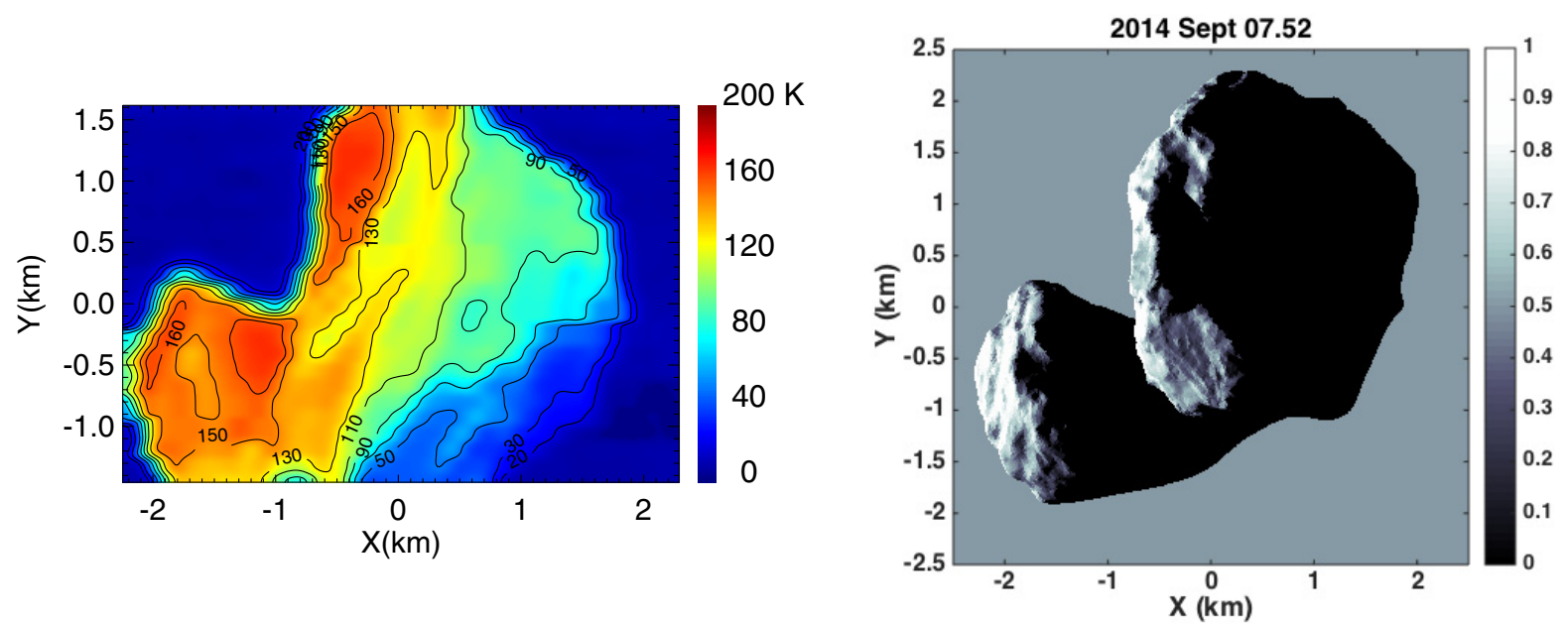

Fig. 1. Left: context submillimeter continuum of the nucleus of $67 \mathrm{P}$ mapped at the same time as the $\mathrm{H}_{2}^{16} \mathrm{O}$ and $\mathrm{H}_{2}^{18} \mathrm{O}$ lines (Figs. 2 and 3). In all maps $X$ and $Y$ refer to the spacecraft coordinates axes, the $X$-axis pointing in the direction of the Sun; the nucleus center of comet $67 \mathrm{P}$ is at the origin of the direction. Intensity contours in brightness temperatures (but assuming $\eta_{\text {fss }}=1.0$ ) are drawn and the color scale is given on the right. Nucleus brightness temperatures vary from $\approx 30 \mathrm{~K}$ in regions of polar night (lower right, Choukroun et al. 2015) to $>160 \mathrm{~K}$ in the warmest regions (left side). As a result of its rapid rotation, the nucleus has been smeared during the three hours of mapping. Right: illumination map of the nucleus of 67P as seen from Rosetta at the central time of the observation (12h30 UT). Map based on the Shape 5 model of 67P (Jorda et al. 2015).

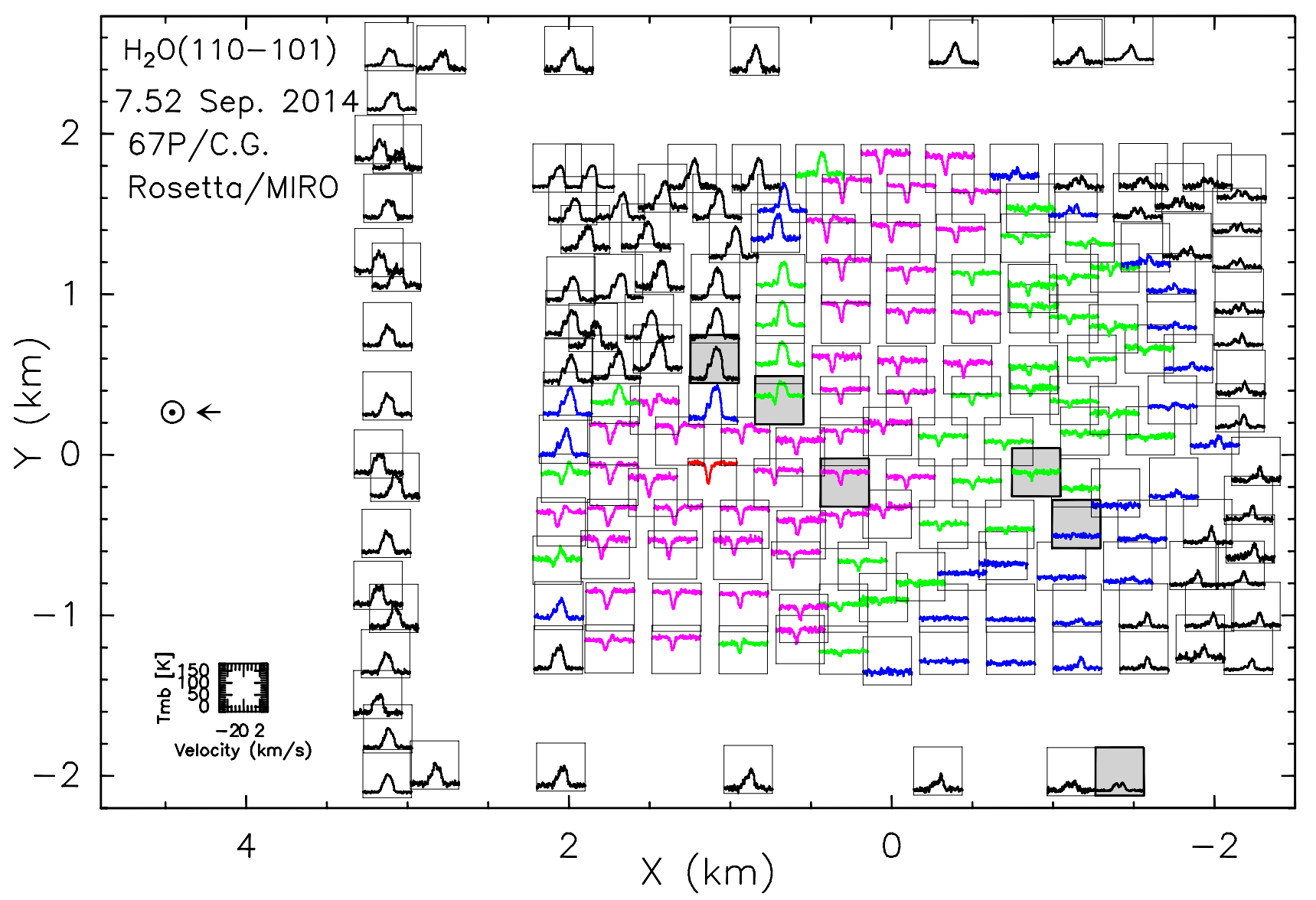

Fig. 2. 201 spectra of the $\mathrm{H}_{2}^{16} \mathrm{O}$ line at $556.936 \mathrm{GHz}$ observed with MIRO between 10h35 and 14h22 UT on 7 September 2014, placed on a map of the sky as seen from the spacecraft with the nucleus center at the origin. Each spectrum corresponds to the average of one to four nearby single spectra (30 to $120 \mathrm{~s}$ integrations) obtained with the MIRO CTS in frequency-switching mode. Each small spectrum is plotted at its mean observed position relative to nucleus center, with the submillimeter continuum added as described in the text. The spectra have been also color coded according to the background submillimeter continuum level: black: $<3 \mathrm{~K}$, blue: $3-50 \mathrm{~K}$, green: $50-100 \mathrm{~K}$, pink: $100-150 \mathrm{~K}$, and red $>150 \mathrm{~K}$. The six selected spectra of Fig. 4 are highlighted. The scales are the same for all spectra: -20 to $+180 \mathrm{~K}$ in $T_{\mathrm{mB}}$ (vertical axis) and -3 to $+3 \mathrm{~km} \mathrm{~s}{ }^{-1}$ in Doppler velocity relative to the nucleus (horizontal axis). The Sun is to the left, the rotation axis north pole points at a PA of $43^{\circ}$ (toward the upper left corner). As discussed in the text (Fig. 4), depending on whether the background is bright or dim relative to the intervening gas, the water line is seen in emission (a peak) or in absorption (a dip), and there are a few intermediate cases. 
a single scan line, and 25 scan lines separated by 7.3' (1 submm beam) were covered sequentially. The full nucleus was covered in three hours, during which it rotated by $\approx 90^{\circ}$ : the visible appearance of the nucleus at mid-point of the map is shown in the right panel of Fig. 1, while its rotation results in a somewhat smeared continuum map, as shown in the left panel of Fig. 1.

In addition, we included in the analysis three straight scans at the beginning and end of the map, at $2.4^{\circ}$ from the center of the nucleus. The total mapping time used is four hours (10.5-14.4 h UT) and the mean distance between the spacecraft and the nucleus center was $58 \mathrm{~km}$, so that the map approximately covers $5 \times 5 \mathrm{~km}$ (Table 1 ). Figure 2 illustrates the results of the mapping with individual spectra of the $\mathrm{H}_{2}^{16} \mathrm{O}$ line at $556.936 \mathrm{GHz}$, averaged into sets of about four nearby integrations to reduce the number of plotted spectra and increase the signal-to-noise ratio. Figure 3 shows the simultaneous map of the $\mathrm{H}_{2}^{18} \mathrm{O}$ line at $547.676 \mathrm{GHz}$ with a much larger binning factor (typically eight $30 \mathrm{~s}$ spectra) because the line is weaker. The background continuum measured with MIRO with the submillimeter continuum channel (Fig. 1) was added to each frequencyswitched and folded $\mathrm{H}_{2}^{16} \mathrm{O}$ spectrum.

\subsection{Calibrating and modeling the MIRO beam}

The MIRO continuum and spectral measurements are calibrated against two onboard targets (Gulkis et al. 2007) and the observed intensities are expressed in antenna temperatures $(\mathrm{Ta})$, which is the Rayleigh-Jeans equivalent temperature that a blackbody filling the MIRO beam would have in order to emit the same amount of thermal energy as is detected by MIRO. The antenna temperatures need to be further corrected for antenna losses because part of the flux is emitted from beyond the main beam and main diffraction pattern of the antenna. We define the main-beam temperature as $T_{\mathrm{mB}}=\frac{T a}{\eta_{\mathrm{mB}}}$ and the temperature measured in the full diffraction pattern as $T_{R *}=\frac{T a}{\eta_{\mathrm{fss}}}$, as is commonly done. $\eta_{\mathrm{mB}}$ is the main-beam efficiency giving the fraction of the flux in the main beam, modeled as a Gaussian of the same full width at half maximum (FWHM) as the real diffraction pattern, and $\eta_{\mathrm{fss}}$ is the fraction of the flux in the whole diffraction pattern (sometimes called moon efficiency), including main beam and side-rings or side-lobes.

The MIRO beam pattern was characterized in the laboratory before flight (Gulkis et al. 2007). According to laboratory measurements of the antenna roughness, the main-beam efficiency at $562 \mathrm{GHz}$ is expected to be about $\eta_{\mathrm{mB}}=0.93$, but 96 to $97 \%\left(\eta_{\mathrm{fss}}\right)$ of the antenna sensitivity is thought to be contained in the full diffraction pattern (main beam and side-lobes), as also suggested by continuum measurements on the comet nucleus (Schloerb et al. 2015). In-flight performance has not yet been determined but cannot be as precisely measured as was pre-flight because we lack a strong astronomical reference source (calibrating the antenna requires a reference outside the instrument). We note, however, that dust in the comet environment striking the antenna might degrade its performance over time.

We modeled the MIRO beam assuming a submillimeter Gaussian beam of $F W H M=562.8 / v(\mathrm{GHz}) \times 456^{\prime \prime}$ plus an error beam $14 \times$ larger that contains $3 \%$ additional flux to simulate a diffraction pattern at submillimeter wavelength that contains about $\eta_{\mathrm{mB}}+3 \%=96 \%$ of the flux $\left(\eta_{\mathrm{fss}}=0.96\right)$. Continuum fluxes were directly converted into the Rayleigh-Jeans temperatures $T_{R *}=\frac{T a}{0.96}$ (and can be further converted to the corresponding blackbody brightness temperatures $T_{\mathrm{B}}$ for physical studies as in Choukroun et al. 2015 and Schloerb et al. 2015). The
Table 1. Observing circumstances.

\begin{tabular}{lccc}
\hline \hline $\begin{array}{l}\text { UT date } \\
\text { (yyyy/mm/dd.d-dd.d) }\end{array}$ & $\begin{array}{c}\left\langle r_{h}\right\rangle \\
(\mathrm{AU})\end{array}$ & $\begin{array}{c}\langle\Delta\rangle \\
(\mathrm{km})\end{array}$ & $\begin{array}{c}\text { Phase angle } \\
\left({ }^{\circ}\right)\end{array}$ \\
\hline $2014 / 09 / 07.44-07.60$ & 3.41 & $59.6-57.0$ & 90.4 \\
\hline
\end{tabular}

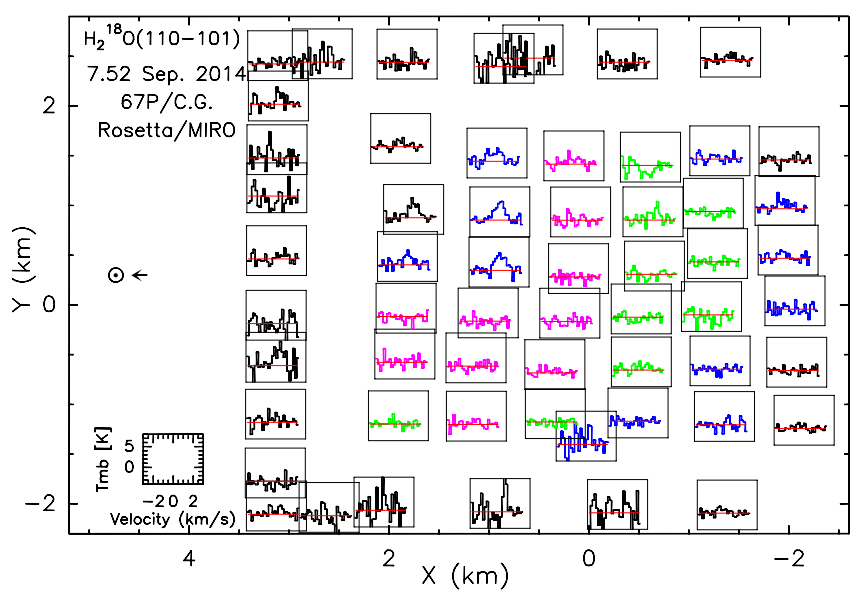

Fig. 3. 58 spectra of the $\mathrm{H}_{2}^{18} \mathrm{O}$ line at $547.676 \mathrm{GHz}$ observed with MIRO between $10 \mathrm{~h} 35$ and $14 \mathrm{~h} 22$ UT on 7 September 2014. This map covers the same observations as in Fig. 2, but with a larger binning of $750 \times$ $510 \mathrm{~m}$. The spectra have been also color coded according to the average background submillimeter continuum level: black: $<3 \mathrm{~K}$, blue: $3-50 \mathrm{~K}$, green: $50-100 \mathrm{~K}$, pink: $100-150 \mathrm{~K}$, and red $>150 \mathrm{~K}$. The line is only clearly seen in emission, while above the neck/subsolar region, $2.0>$ $X>0.5 \mathrm{~km}$ and $1.5>Y>0 \mathrm{~km}$ The scales are the same for all spectra: -4 to $+8 \mathrm{~K}$ in $T_{\mathrm{mB}}$ (vertical axis) and -3 to $+3 \mathrm{~km} \mathrm{~s}^{-1}$ in Doppler velocity relative to the nucleus (horizontal axis).

frequency-switched spectra show the line in negative at $-5 \mathrm{MHz}$ and positive at $+5 \mathrm{MHz}$. They were folded to increase the signalto-noise ratio (by $\sqrt{2}$ ) and were then converted into $T_{\mathrm{mB}}=\frac{T a}{0.93}$ to be compared to the simulation (main beam + error beam).

\section{Model assumption and data analysis}

\subsection{Sample spectra and various configurations}

Figure 4 shows several spectra taken approximately along the projection of the rotation axis from the dayside of the coma to the nightside, crossing the illuminated and nightside part of the nucleus. Their positions are highlighted in Fig. 2. They illustrate the different cases we have to analyze:

1. strong line in emission in the dense coma on the dayside;

2. line partly in emission, partly in absorption because the beam sampled both nucleus and coma on the limb;

3. line in absorption against warm illuminated nucleus;

4. line in absorption against cool nucleus;

5. weak line in emission against the cold dark nucleus; and

6. line in emission on the nightside of the coma.

\subsection{Model parameters}

In cases (1), (3), (4), (5), and (6), the background continuum $T_{R *}$ is derived from the simultaneous observation with the submm continuum channel. But for case (2), the continuum level in the beam is due to incomplete filling of the beam by the nucleus surface, and we took the nucleus continuum from nearby measurements fully on the nucleus and adjusted the local nucleus 


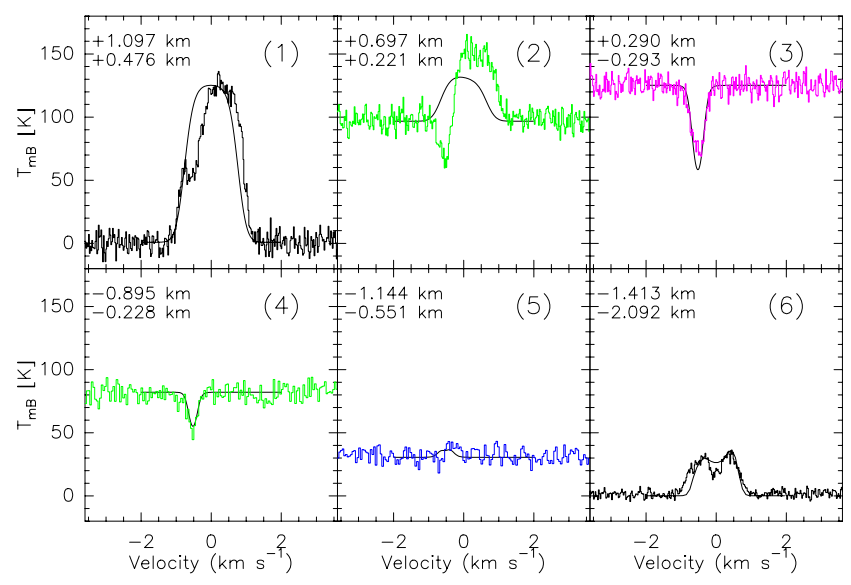

Fig. 4. Six spectra of the $\mathrm{H}_{2}^{16} \mathrm{O}$ line at $556.936 \mathrm{GHz}$ observed with MIRO extracted from the map in Fig. 2, approximately along the projected rotation axis of comet 67P. The pointing offsets in meters relative to the direction of the center of the nucleus are given in the upper left corner of each spectrum. The continuum level has been added and color coded as in Fig. 2. The six spectra labeled (1)-(6) illustrate the corresponding cases in Sect. 3. We note that in the cases (3)-(5) the line is either weakly in emission or in absorption, but only at negative velocities, i.e., coming from gas moving toward Rosetta/MIRO, because the nucleus shields the coma emission from the far side of the comet. The scales are the same for all spectra: -20 to $+180 \mathrm{~K}$ in $T_{\mathrm{mB}}$ (vertical axis) and -3.6 to $+3.6 \mathrm{~km} \mathrm{~s}^{-1}$ in Doppler velocity relative to the nucleus (horizontal axis). Simulated profiles (smooth black curves) obtained with the parameters of Table 2 are superimposed.

radius at the considered offset position to match the observed continuum level.

\subsubsection{Gas velocity and outgassing pattern}

To simplify the analysis and reduce the free parameters, we assumed radial outflow at a constant velocity $v_{\exp }$ and a Haser radial density distribution. For lines in absorption (case 3 and 4), $v_{\text {exp }}$ is the value of the Doppler-shift of the peak absorption. A Gaussian fit to the high spectroscopic resolution lines obtained with MIRO provides the value for $v_{\text {exp }}$. The subsolar nadir observations of August (Gulkis et al. 2015; Lee et al. 2015) gave a value $v_{\exp } \approx 0.70 \mathrm{~km} \mathrm{~s}^{-1}$. The nadir pointings in this map (phase angle is $90^{\circ}$ ) give lower absolute values from 0.47 to $0.59 \mathrm{~km} \mathrm{~s}^{-1}$ (see also Fig. 6 right). For nadir pointing we used a single value, $v_{\text {exp }}=0.52 \mathrm{~km} \mathrm{~s}^{-1}$, similar as for nightside weaker emissions (cases (5) and (6)). Since a 10-20\% error in velocity will result in similar errors in the production rate and smaller errors in column density, using the fixed value $0.52 \mathrm{~km} \mathrm{~s}^{-1}$ instead of the result of the value of $v_{\text {exp }}$ inferred from each spectrum will not produce a large bias. The value of $v_{\exp }$ we used is the velocity of the bulk of the gas along the line of sight, close to the terminal velocity after acceleration in the first meters to kilometers above the surface of the nucleus. Our code can simulate this variable velocity (Biver et al. 2011), but this would add additional parameters and requires too much time to constrain them for the accuracy targeted here.

We used 1D (radial) modeling of the density and molecular excitation, but we restricted the density to a non-zero value in a limited solid angle $\Omega$ given by a range in azimuth $\theta$ and colatitude $\phi$ in spherical coordinates, with the main axis on the line of sight. For nadir viewing, that is, when the nucleus fills the beam and we cannot see gas beyond the nucleus, we should

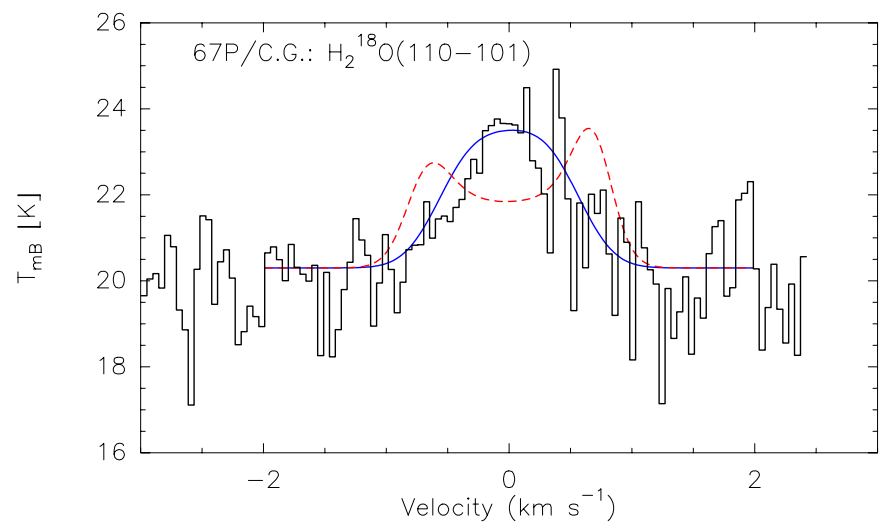

Fig. 5. Spectrum of $\mathrm{H}_{2}^{18} \mathrm{O}$ from averaging the four strongest emission lines in the pole region of Fig. 3. The line width $(1.01 \pm$ $\left.0.1 \mathrm{~km} \mathrm{~s}^{-1}\right)$ is lower than expected for isotropic outgassing at $v_{\exp }=$ $0.70 \mathrm{~km} \mathrm{~s}^{-1}$ ( $F W H M=1.36 \mathrm{~km} \mathrm{~s}^{-1}$ with a double peak, dashed line). If we assume that most of the outgassing is limited to a restricted cone $\left(\phi=90 \pm 48^{\circ}, \theta= \pm 48^{\circ}\right.$, see text), we obtain a reasonable agreement to the line shape (superimposed continuous profile, with $Q_{\mathrm{H}_{2}^{18} \mathrm{O}}=1.26 \times 10^{23} \mathrm{molec} \mathrm{s}^{-1}$ ). Part of the background continuum of the nucleus is also seen and modeled.

restrict $\phi<90^{\circ}\left(-180<\theta<+180^{\circ}, \Omega=2 \pi\right)$ or less, but the derived column density or production rate per solid angle does not really depend on the assumed width $\Delta \phi$ of the cone of outgassing $\Omega$.

For off-limb observations, we also considered hemispheric outgassing $(\Omega=2 \pi)$ with the line of sight fully contained in that hemisphere. On the sunward side, the water line is saturated, but the $\mathrm{H}_{2}^{18} \mathrm{O}$ line (Fig. 5) is detected and optically thin. In this case, the $\mathrm{H}_{2}^{18} \mathrm{O}$ line width (Fig. 5) suggests that the bulk of the outgassing is in a more restricted region of the sky, and we assumed a $\Omega=0.80 \pi$ "jet" $\left(\phi=90 \pm 48^{\circ}, \theta= \pm 48^{\circ}\right)$. The measured FWHM $\left(1.01 \pm 0.1 \mathrm{~km} \mathrm{~s}^{-1}\right)$ of the line is compatible with halfopening angles $(\Delta \phi \approx \theta)$ of the jet in the range $36-47^{\circ}$, while the $\mathrm{H}_{2}^{16} \mathrm{O}$ line (Fig. 4-(1)) suggests slightly higher values.

\subsubsection{Temperature profile}

Hydrodynamical models (Zakharov, priv. comm.; Zakharov et al. 2007) predict that the kinetic gas temperature decreases from $\approx 0.86 \times T_{\text {surf }}$ at the nucleus surface to very low temperatures as the radial distance $r$ increases. This behavior is expected from the adiabatic cooling of the gas that expands into vacuum. The decrease of the peak intensity of the optically thick water line (in emission off-nucleus) has been observed by MIRO and confirms this expected behavior. We modeled the kinetic gas temperature following $T=T_{0} \times\left(0.66 \times\left(r / r_{\text {nuc }}\right)+0.20\right)$, with $r_{\text {nuc }}=2.0 \mathrm{~km}$, and $T=0.86 \times T_{0}$ locally where the limb is closer than $2 \mathrm{~km}$ from the center of the nucleus. On the dayside, the saturated water line just off-nucleus peaks at $T_{\mathrm{mB}} \approx 132 \mathrm{~K}$ ((1) in Fig. 4). Using $T_{0}=165 \mathrm{~K}$ with our full non-local thermodynamical equilibrium (non-LTE) model yields a good fit to the peak line intensity.

Since it is beyond the scope of this paper to retrieve the temperature profile in each point, we tried to use the smallest number of parameter sets (Table 2) and thereby avoided other uncertainties and extensive computation time that can rise from the use of inversion codes. We found evidence for lower initial temperatures on the nightside (cool side): the transition from case (3) to (4) is seen for a background continuum in the range 
Table 2. Set of parameters for the analysis.

\begin{tabular}{ccccccc}
\hline \hline Case & \multicolumn{2}{c}{$\begin{array}{l}\text { Geometry }- \text { jet } \\
\theta\end{array}$} & $\Omega$ & $\begin{array}{c}v_{\text {exp }} \\
\left(\mathrm{km} \mathrm{s}^{-1}\right)\end{array}$ & $\begin{array}{c}T_{\text {bg }} \\
(\mathrm{K})\end{array}$ & $\begin{array}{c}T_{\text {kin }} \\
(\mathrm{K})\end{array}$ \\
\hline 1 & $52-138^{\circ}$ & $\pm 48^{\circ}$ & $0.8 \pi$ & 0.70 & 2.73 & $165 \times\left(0.66 \times\left(r / r_{\text {nuc }}\right)+0.20\right)$ \\
2 & \multicolumn{2}{c}{ cases $1+3$} & $1.6 \pi$ & $0.52,0.7$ & $T_{\text {nearby-nuc }}$ & $165 \times\left(0.66 \times\left(r / r_{\text {nuc }}\right)+0.20\right)$ \\
3 & $0-53^{\circ}$ & $\pm 180^{\circ}$ & $0.8 \pi$ & 0.52 & $T_{\text {nuc }}$ & $T_{\text {nuc }} \times\left(0.66 \times\left(r / r_{\text {nuc }}\right)+0.20\right)$ \\
4 & $0-53^{\circ}$ & $\pm 180^{\circ}$ & $0.8 \pi$ & 0.52 & $T_{\text {nuc }}$ & $T_{\text {nuc }} \times\left(0.66 \times\left(r / r_{\text {nuc }}\right)+0.20\right)$ \\
5 & $0-53^{\circ}$ & $\pm 180^{\circ}$ & $0.8 \pi$ & 0.52 & $T_{\text {nuc }}$ & $80 \times\left(0.66 \times\left(r / r_{\text {nuc }}\right)+0.20\right)$ \\
6 & $0-180^{\circ}$ & $\pm 90^{\circ}$ & $2 \pi$ & 0.52 & 2.73 & $100 \times\left(0.66 \times\left(r / r_{\text {nuc }}\right)+0.20\right)$ \\
\hline
\end{tabular}
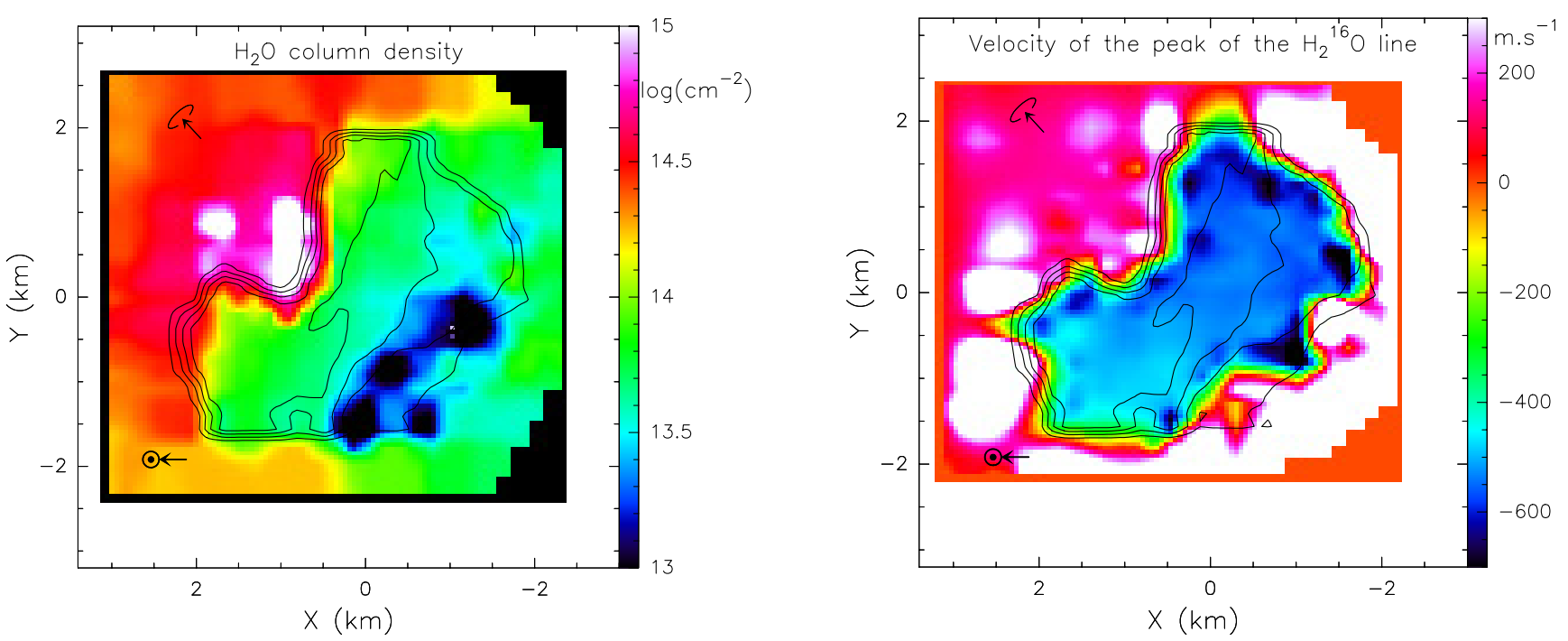

Fig. 6. Left: Log scale plot of the derived water column density around the nucleus of 67P. Color-coded scale in $\log \left(\operatorname{molec} \mathrm{cm}^{-2}\right)$ is given at the right. Right: values of the peak velocity of the $\mathrm{H}_{2}^{16} \mathrm{O}$ line (from Gaussian-fitting the strongest component). Negative velocities, seen against the nucleus from lines in absorption, indicate a flow towards the observer. Off the nucleus, the line is mostly redshifted (positive velocities) as a result of self-absorption of the foreground part of the coma in the optically thick $\mathrm{H}_{2}^{16} \mathrm{O}$ line, which causes gas moving away from us on the far side of the nucleus to dominate the spectrum. Contours of the continuum level (in black), locating the nucleus, are superimposed. The direction of the Sun and north pole rotation axis are also shown.

of 30-45 K. A temperature profile with $T_{0}=80 \mathrm{~K}$ yields a transition from the line in absorption to emission for this range of background nucleus temperature. We therefore used $T_{0}=80 \mathrm{~K}$, or the measured background temperature $T_{\mathrm{B}}$ if this was higher, to analyze the lines in cases (3) to (5). For type (6) we fixed $T_{0}=100 \mathrm{~K}$. Table 2 provides a summary of the various parameter sets.

The simulations corresponding to the six observations, cases (1)-(6), are superimposed in Fig. 4. The fit is not perfect, but good enough to obtain a reasonable order of magnitude for the column density.

\section{Water column density around the nucleus}

\subsection{Computing column densities and production rates}

Our code accounts for a non-LTE time variable evolution of the rotational population of the $\mathrm{H}_{2}^{16} \mathrm{O}$ or $\mathrm{H}_{2}^{18} \mathrm{O}$ molecules as they move away from the nucleus. Collisions with neutrals, electrons, and infrared radiative pumping was taken into account as described in Zakharov et al. (2007). Optical effects using the escape probability method for excitation and for radiative transfer were also taken into account.

The density profile was modeled assuming a Haser radial density profile in a restricted angular region. For each point we assumed a locally spherical nucleus with a local radius $r_{\text {nuc }}$ ( 0.75 to $2.32 \mathrm{~km}$ ) compatible with the observations. We used the pointing offset relative to the center of the nucleus. When we pointed outside the nucleus or fully on the nucleus, the assumed radius value does not matter. When we pointed on the limb, we adjusted the value of $r_{\text {nuc }}$ in the code to find the proper filling of the beam that yields the observed continuum.

When the density is high, especially on the dayside, deriving the water column density from the $\mathrm{H}_{2}^{16} \mathrm{O}$ line is difficult. The $556.936 \mathrm{GHz}$ line is optically thick, and its intensity is much more sensitive to the gas temperature, velocity profile, and outgassing pattern than global production. We scaled the densities using the $\mathrm{H}_{2}^{18} \mathrm{O}$ line, assuming $\mathrm{H}_{2}^{16} \mathrm{O} / \mathrm{H}_{2}^{18} \mathrm{O}=500$, when detected. In all comets (e.g., Biver et al. 2007; Bockelée-Morvan et al. 2012), including 67P as measured by the ROSINA instrument (Altwegg et al. 2015), the $\mathrm{H}_{2}^{16} \mathrm{O} / \mathrm{H}_{2}^{18} \mathrm{O}$ ratio is within $10 \%$ of the terrestrial value (499). Assuming $\mathrm{H}_{2}^{16} \mathrm{O} / \mathrm{H}_{2}^{18} \mathrm{O}=500$ will not introduce an error larger than $10 \%$.

For each spectrum we estimated the corresponding water column density and production rate per solid angle. Figure 6 displays the resulting map of the column density in a color-coded way.

\section{Distribution of water production around the nucleus}

We clearly see evidence from Fig. 6 that the gas is nonuniformly spread around the nucleus. Column densities are more 


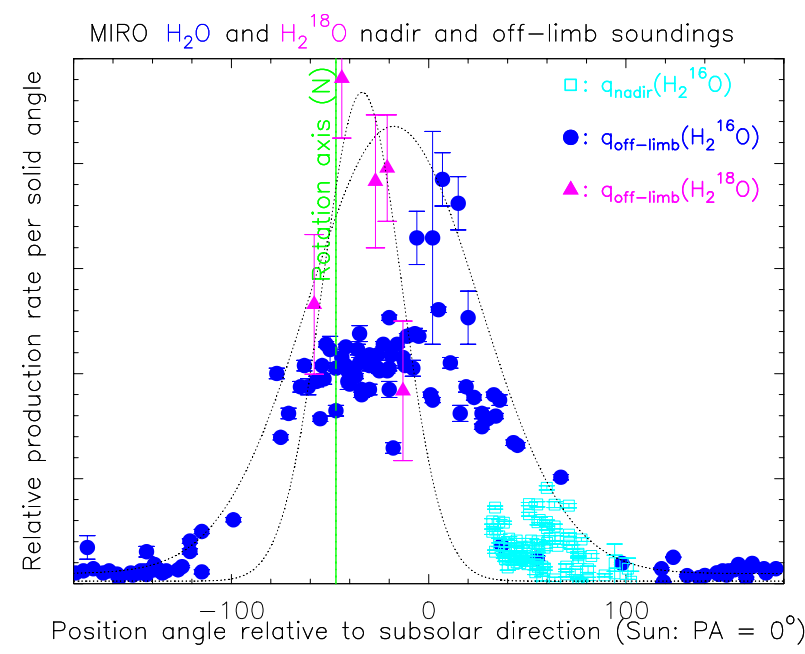

Fig. 7. Production rates per solid angle derived for each point versus their angular position in the plane of sky relative to the direction of the Sun. For measurements against the nucleus, we have determined a rough position angle between the plane containing the targeted point (assumed to be at $2 \mathrm{~km}$ from the center) and the terminator and the plane of the sky. These nadir-directed points are shown as light squares. There is a clear peak in emission between angles of -100 and $+60^{\circ}$, forming a broad jet from a region on the nucleus including the subsolar point and the north pole. Independent Gaussian fits to the $\mathrm{H}_{2}^{16} \mathrm{O}$ and $\mathrm{H}_{2}^{18} \mathrm{O}$ data are plotted as dotted lines. The vertical scale for $\mathrm{H}_{2}^{16} \mathrm{O}$ has been multiplied by 125 relative to $\mathrm{H}_{2}^{18} \mathrm{O}$ for legibility, but we would expect a factor $\approx 4$ more (matching the expected ${ }^{16} \mathrm{O} /{ }^{18} \mathrm{O}$ ratio of 500 ) with a better modeled opacity. The $\mathrm{H}_{2}^{16} \mathrm{O}$ production rates (blue dots and squares) are very likely underestimated because the line is not fully modeled due to saturation (Fig. 4-(1)) in the region -70 to $0^{\circ}$, which explains the broader angular shape. Nadir points (squares) suggest that the jet probably is narrower in the plane of the line of sight and Sun direction.

two orders of magnitude higher near the "neck" region (direction of rotation axis) than against the polar night part of the nucleus.

We converted these column densities into the radial outgassing rate per unit solid angle. Figure 7 shows these values plotted as a function of the angle on the plane of sky from the direction of the Sun, and of the angle relative to the plane of sky for nadir pointings. For reasons discussed in Sect. 6.1, the vertical scale should be considered uncertain, although the shape of the curve is more reliable. Figure 7 shows that the bulk of the outgassing is limited to a relatively narrow angle close to a direction between the rotation axis and the Sun. The shape can be approximated by a low uniform background at $q_{\mathrm{bg}} \approx 1.5 \times 10^{23}$ molec s$^{-1} \mathrm{sr}^{-1}\left(Q_{\mathrm{bg}} \approx 0.2 \times 10^{25}\right.$ molec s$\left.^{-1}\right)$ and a Gaussian with $F W H M \approx 80^{\circ}$ centered halfway between the directions to the north pole and the Sun. The corresponding solid angle would be $\Omega=0.6 \times \pi$, so that the total outgassing rate is $Q=2 \times q_{\max }$, where $q_{\max }$ is the peak angular production rate. This agrees with our findings based on the $\mathrm{H}_{2}^{18} \mathrm{O}$ line shape and the jet modeling with a full width angle of $72-96^{\circ}$ (Sect. 3.2.1, Fig. 5).

\section{Discussion}

\subsection{Uncertainty on column densities and outgassing rates}

Deriving precise quantitative information from the optically thick $\mathrm{H}_{2}^{16} \mathrm{O}$ line is beyond the scope of this paper. When the line is close to saturation, which is the case on the dayside, the peak intensity strongly depends on the distribution of the gas temperature along the line of sight. It becomes nonlinearly dependent on the density or assumed production rate per unit angle. The radial temperature profile is obviously not the same in all space directions, which means that we certainly do not model the coma precisely. We are encouraged, however, because Fig. 7 shows that for a given radial vector (same position angle) the scatter in $q_{\mathrm{H}_{2} \mathrm{O}}$ is smaller than the jet feature of interest. But there is still a discrepancy between the production rates based on $\mathrm{H}_{2}^{16} \mathrm{O}$ and $\mathrm{H}_{2}^{18} \mathrm{O}$, probably because we systematically underestimate the $\mathrm{H}_{2}^{16} \mathrm{O}$ line opacity as we assumed uniform density in the cone, while it is certainly more locally concentrated (e.g., the angular density distribution profile is more Gaussian than flat). This effect does not strongly affect the optically thin $\mathrm{H}_{2}^{18} \mathrm{O}$ line, so it can be used for more quantitative measurements, but the lower signal-to-noise ratio of the $\mathrm{H}_{2}^{18} \mathrm{O}$ line creates some uncertainty as well.

\subsection{Effect of rotation of the nucleus during mapping}

Given the $12.4 \mathrm{~h}$ rotation period of the nucleus of comet $67 \mathrm{P}$, the nucleus rotated by $90^{\circ}$ during the mapping. Since the scanning was made from south to north, as the nucleus rotation moved the lower part up, we rather oversampled the central part of the nucleus. But regarding the coma in addition to the nucleus, we are sampling a much broader azimuth range. We do not aim for a very detailed view of the water distribution around the nucleus because we did not consider topographical effects (e.g., local slopes) in this paper. We are confident in the reality of the broad coma features derived in this analysis, in spite of the rotational smearing.

\subsection{Nightside outgassing or backflow from dayside?}

The $\mathrm{H}_{2}^{16} \mathrm{O}$ line is detected at all 201 points, except at five points against the cold dark nucleus $\left(T_{\mathrm{B}}=40-70 \mathrm{~K}\right)$. These nondetections might be due to too high noise (short integration times) and too low contrast between the gas and background temperatures. Thus, there may be water on all lines of sight. But even if true, it is possible that there is virtually no emission from the nightside nucleus, and the water seen there originates in a nonradial flow from the dayside.

The shape of the water line observed against the nucleus might help distinguish between local outgassing and water flowing back from the region of high production on the dayside. Figure 6, right, shows the Doppler shift of the peak of absorption or emission of the $\mathrm{H}_{2}^{16} \mathrm{O}$ line. Across most of the nucleus we find the same value (close to $-0.52 \mathrm{~km} \mathrm{~s}^{-1}$ as discussed previously), slightly higher $\left(-0.60 \mathrm{~km} \mathrm{~s}^{-1}\right)$ toward the upper part, but no day-night trend. If the radial outflow were at a lower speed in colder regions or if there were a flow from the dayside with a stronger projection effect as we move toward the nightside, we would expect a decreasing peak velocity when moving from day- to nightside. We do not see this, therefore we believe there is local emission from the nightside. Nevertheless, lower (absolute) velocities in the bottom part (small lobe) and even some redshifted emission in the lower right part might indicate that there is a larger contribution from a nonradial flow against this part of the comet.

Other evidence that there is some backflow of water from day- to nightside (even if not dominant close to the nucleus) is the clear detection of water emission (Figs. 2 and 6) beyond the nucleus on the nightside at levels that are often higher than against the nucleus. This suggests that we do have a contribution 
that is due to gas flowing back to the nightside, although the column densities are much lower than on the day side.

To summarize this discussion, we conclude that nightside emission from the nucleus can be an important source of water vapor in the nightside coma near the nucleus, but that there is also clear evidence for another source, probably the expected dayside to nightside flow within the coma. A more detailed analysis of MIRO data, using more complex 2D or 3D coma models, should allow a quantifiable analysis of this in the future.

\subsection{Conclusions}

The MIRO instrument has been mapping the water coma of comet 67P, and we here presented our first detailed analysis of early measurements, made in September 2014 at a heliocentric distance of $3.4 \mathrm{AU}$. In this data set we can globally distinguish three parts of the coma, based on column densities:

- The highest column density is seen in the neck to subsolar region and is 1-2 orders of magnitude higher than elsewhere. Globally, outgassing can be modeled as concentrated in a cone with an azimuthal Gaussian density profile of $F W H M \approx 80 \pm 20^{\circ}$ corresponding to $\Omega=0.6 \pm 0.3 \times \pi$ sr. The concentration of water vapour in this region might suggest that outgassing activity is not only related to illumination (Fig. 1 right), but that topography also certainly plays an important role in focusing the expansion of the gas in a preferred direction.

- Significant outgassing with a density about 10-20× lower is observed up to the terminator $\left(80-90^{\circ}\right.$ from either pole or subsolar point); this region is included in the Gaussian profile mentioned above.

- Very low outgassing ( $<1 \%$ of the peak) or possibly backflow is seen on the nightside. This component contributes with $\approx 0.2 \times 10^{25}$ molec s$^{-1}$ to our estimated total outgassing rate, which is less than $10 \%$ of the comet's total water production (see next paragraph).

We consequently may infer an estimate of the total outgassing rate from the peak outgassing per solid angle of $\mathrm{H}_{2}^{18} \mathrm{O}\left(q_{\mathrm{H}_{2}^{18} \mathrm{O}} \approx\right.$ $5 \pm 1 \times 10^{22}$ molec s $\left.{ }^{-1} \mathrm{sr}^{-1}\right)-$ not affected by opacity effects - multiplied by 500 and $\Omega$ (and adding the low-level outgassing from other areas mentioned above). This yields $Q_{\mathrm{H}_{2}^{16} \mathrm{O}} \approx 4.9 \pm$ $2.5 \times 10^{25}$ molec s $^{-1}$ for the 7.5 September 2014 observations at $r_{h}=3.41 \mathrm{AU}$ from the Sun.

MIRO continues to map the nucleus and coma, and eventually, we will have observations of the evolution of the coma as the comet moves through perihelion and beyond. These first results demonstrate the usefulness of MIRO for understanding conditions and processes at work in the nucleus and coma of a comet.

Acknowledgements. The authors acknowledge support from their institutions and funding sources. A part of the research was carried out at the Jet Propulsion Laboratory, California Institute of Technology, under a contract with the National Aeronautics and Space Administration. A part of the research was carried out at the Max-Planck-Institut für Sonnensystemforschung with financial support from Deutsches Zentrum für Luft- und Raumfarht and Max-PlanckGesellschaft. Parts of the research were carried out by LESIA and LERMA, Observatoire de Paris, with financial support from CNES and CNRS/Institut des Sciences de l'Univers. A part of the research was carried out at the National Central University with funding from the Taiwanese National Science Counsel grant NSC 101-2111-M-008-016. A part of the research was carried out at the University of Massachusetts, Amherst, USA. We thank Y. Anderson, T. Koch, R. Nowicki, L. Pan and the late Lucas Kamp for their efforts in scheduling, operations, and support of the MIRO instrument.

\section{References}

Altwegg, K., Balsiger, H., Bar-Nun, A., et al. 2015, Science, 347, 1261952 Biver, N., Bockelée-Morvan, D., Crovisier, J., et al. 2007, Planet. Space Sci., 55, 1058

Biver, N., Bockelée-Morvan, D., Colom, P., et al. 2011, A\&A, 528, A142

Bockelée-Morvan, D., Biver, N., Swinyard, B., et al. 2012, A\&A, 544, L15

Choukroun, M., Kheim, S., Schloerb, F.P., et al., 2015, A\&A, 583, A28

Gulkis, S., Frerking, M., Crovisier, J., et al. 2007, Space Sci. Rev., 128, 561

Gulkis, S., Allen, M., von Allmen, P., et al. 2015, Science, 347, aaa0709

Hartogh, P., \& Hartmann, G. K. 1990, Meas. Sci. Technol., 1, 592

Hartogh, P., Crovisier, J., de Val-Borro, M., et al. 2010, A\&A, 518, L150

Hartogh, P., Lis, D. C., Bockelée-Morvan, D., et al. 2011, Nature, 478, 218

Jorda, L., Gaskell, R., Hviid, S. L., et al. 2015, NASA Planetary Data System and ESA Planetary Science Archive

Lee, S., von Allmen, P., Allen, M., et al. 2015, A\&A, 583, A5

Lecacheux, A., Biver, N., Crovisier, J., et al. 2003, A\&A, 402, L55

Neufeld, D. A., Stauffer, J. R., Bergin, E. A., et al. 2000, ApJ, 539, L15

Schloerb, F. P., Keihm, S., von Allmen, P., et al. 2015, A\&A, 583, A29

Zakharov, V., Bockelée-Morvan, D., Biver, N., Crovisier, J., \& Lecacheux, A. 2007, A\&A, 473, 303 